\title{
Small-action particles in a tokamak in the presence of an $n=1$ mode
}

\author{
Ya. I. Kolesnichenko*, V. V. Lutsenko*, R. B. White**, and Yu. V. Yakovenko*, \\ *Scientific Centre "Institute for Nuclear Research", Kyiv, 252680, Ukraine \\ ** Princeton Plasma Physics Laboratory, P.O.Box 451, Princeton, NJ, 08543, USA
}

(October 19, 1999)

\begin{abstract}
It is found that an $m=n=1$ mode with the amplitude exceeding a certain threshold can lead to stochastic motion of energetic ions in tokamaks, the large orbit width particles (potatoes) being most easily affected. An $n=1$ mode can redistribute particles also in the absence of stochasticity but only when the perturbation is quickly switched on/off, e.g., due to sawtooth crash. In the latter case, the perturbation results in regular motion of particles around a certain helical orbit, at which a resonance driven by the mode but having no amplitude threshold takes place.
\end{abstract}

Most energetic ions that are present in tokamak plasmas are located in the plasma core. Their orbits lying inside the radius $r \sim \nu(\mathcal{E}) A a\left[\nu=\left(2 q_{0} \rho / k R\right)^{2 / 3} ; \rho=v / \omega_{B} ; v=(2 \mathcal{E} / M)^{1 / 2}\right.$; $\mathcal{E}, M$, and $\omega_{B}$ are the particle energy, mass, and gyrofrequency, respectively; $q_{0}$ is the central safety factor; $a, R$, and $A$ are the minor radius, major radius, and aspect ratio of the torus, respectively; $k$ is the ellipticity of the plasma cross section] are characterized by the potatolike or approximately circular shape in the poloidal cross section. These particles may have the orbit width of the order of the mentioned region, but there is also a small group with the orbits having the vanishing width, so-called "stagnation orbits". A distinctive feature of particles in the region $r / a \lesssim \nu(\mathcal{E}) A$ is that they are characterized by the poloidal action integral $J_{\theta} \equiv \oint d \vartheta P_{\vartheta} / 2 \pi\left(P_{\vartheta}\right.$ is the poloidal canonical momentum [1], $\vartheta$ is the poloidal angle) which is small compared to that of the narrow-orbit circulating and trapped (banana-like) particles, tending to zero when the orbit approaches the stagnation orbit. Therefore, we will refer to potatoes and stagnation particles as Small Action Particles (SAP). The small action energetic ions (we are interested only in such SAP's) typically occupy the region inside the $q=1$ radius, which is often characterized by the low- $n$ MHD activity of a plasma $(n$ is the toroidal mode number $)$. The SAP's and low- $n$ perturbations $\propto \exp (-i \omega t+i m \vartheta-i n \varphi)$ with the frequency $\omega \ll \omega_{b}$ ( $\omega_{b}$ is the bounce frequency) may interact through the resonance $s \omega_{b}=n \omega_{\varphi}\left(\omega_{\varphi}\right.$ is the precession frequency in absence of a perturbation, $s$ is an integer) for variety of $s$. In contrast to this, the large-action particles, such as narrow bananas, can interact with the mentioned modes only through the $s=0$ resonance. The difference arises because $\omega_{b} \gg \omega_{\varphi}$ for bananas, whereas $\omega_{b} \gtrsim \omega_{\varphi}$ for SAP's. The resonance interaction of SAP's with the $n=2$ and $n=3$ modes was considered in Ref. [2], where it was shown that such an interaction can essentially redistribute the energetic ions. However, no published works consider the effect of the $n=1$ mode, which is often dominant in MHD events (in, e.g., sawtooth and fishbone oscillations).

In this Letter we study the influence of the $m=n=1$ mode with $\omega \ll \omega_{b}$ on the small-action energetic ions. We show that, in spite of the fact that $\omega_{b} \neq \omega_{\varphi}$, the mode may interact with SAP's resonantly, resulting in their redistribution in a plasma. In particular, we find that finite amplitude of the harmonic perturbation leads to appearance of toroidal mode numbers exceeding unity in action-angle variables of the perturbed system. This produces driven resonance islands which may cause stochastic motion of particles, first of all, of potatoes. We also find that a sharply appearing/disappearing perturbation (e.g., a sawtooth crash) can result in particle redistribution even in the absence of stochasticity, this effect having no amplitude threshold.

To analyze the particle guiding-center motion, we use action-angle variables of the unperturbed system, proceeding from the following Hamiltonian:

$$
H\left(J_{\theta}, J_{\phi}, \theta, \phi\right)=H^{(0)}\left(J_{\theta}, J_{\phi}\right)+V\left(J_{\theta}, J_{\phi}, \theta, \phi\right),
$$


where $H^{(0)}$ is the Hamiltonian of the motion in the absence of the perturbation; $\theta=\theta(\vartheta)$ and $\phi=\varphi+\chi(\vartheta)$ are the canonical poloidal and toroidal angles [3,2]; $\chi(\vartheta)$ is a periodic function; $J_{\theta}$ and $J_{\phi} \equiv \oint d \varphi P_{\varphi} / 2 \pi$ are the corresponding action variables; $P_{\varphi}$ is toroidal canonical momentum; $V=\sum_{s} V_{s}\left(J_{\theta}, J_{\phi}\right) \cos (s \theta-\phi-\omega t)$ is an $n=1$ perturbation. The existence of many harmonics in $\theta$ is a consequence of the toroidicity of the system resulting in non-uniformity of the particle motion in the poloidal direction. This should not be confused with MHD mode coupling due to toroidicity, which often leads to appearance of the sidebands with the poloidal mode number $m+1$ and $m-1$ and the amplitude $\sim A^{-1}$. The non-uniformity of the poloidal motion of trapped particles is $\sim 1$; therefore, the Fourier spectrums of a perturbation in $\vartheta$ and $\theta$ are completely different. In particular, quasi-stagnation particles almost do not "feel" the poloidal (in $\vartheta$ ) structure of the mode. For such particles, the harmonics with $s=0$ and $s=1$ dominate.

At first, we restrict ourselves to the system linearized near $J_{\theta}=0$, taking $H^{(0)}=\omega_{\theta} J_{\theta}+\omega_{\phi} J_{\phi}$, where $\omega_{\theta}=\omega_{b}$ and $\omega_{\phi}=\omega_{\varphi}$ are the frequencies of the unperturbed motion, and consider only the $s=0$ and $s=1$ harmonics of the perturbation. We take $V_{0}=$ const and $V_{1}=$ $\alpha J_{\theta}^{1 / 2}$. The latter can be justified as follows. First, the perturbed part of Hamiltonian is expected to be proportional to the generalized coordinates. Second, an action $J=\oint p d q$ is proportional to the square of the contour size when the contour shrinks to a point. The simplified system is integrable. Performing the canonical transformation $\hat{\theta}=\theta, \hat{\phi}=\theta-\phi-\omega t$, $\hat{J}_{\theta}=J_{\theta}+J_{\phi}+V_{0} \omega_{\phi}^{-1} \cos (\phi+\omega t), \hat{J}_{\phi}=-J_{\phi}-V_{0} \omega_{\phi}^{-1} \cos (\phi+\omega t)$, we obtain the following new Hamiltonian:

$$
\begin{aligned}
\hat{H}\left(\hat{J}_{\theta}, \hat{J}_{\phi}, \hat{\theta}, \hat{\phi}\right) & =\hat{\omega}_{\theta} \hat{J}_{\theta}+\hat{\omega}_{\phi} \hat{J}_{\phi} \\
& +\alpha\left(\hat{J}_{\theta}+\hat{J}_{\phi}\right)^{1 / 2} \cos \hat{\phi}
\end{aligned}
$$

where $\hat{\omega}_{\theta}=\omega_{\theta}, \hat{\omega}_{\phi}=\omega_{\theta}-\omega_{\phi}+\omega$. As $\partial \hat{H} / \partial \hat{\theta}=0, \hat{J}_{\theta}$ is a constant of motion. The level contours of the Hamiltonian (2) for a given $\hat{J}_{\theta}$ are shifted circles in the coordinates $\left(\hat{J}_{\phi}-\hat{J}_{\phi 0}\right)^{1 / 2} \cos \hat{\phi}$, $\left(\hat{J}_{\phi}-\hat{J}_{\phi 0}\right)^{1 / 2} \sin \hat{\phi}$, where $\hat{J}_{\phi 0}=-\hat{J}_{\theta}$ is the value of $\hat{J}_{\phi}$ that corresponds to $J_{\theta}=0$, i.e., the stagnation orbit. Note that $\hat{J}_{\phi}-\hat{J}_{\phi 0}=J_{\theta}$. The center of the shifted circles is located at the fixed point of the Hamiltonian, $J_{\theta}^{1 / 2}=\alpha /\left(2 \hat{\omega}_{\phi}\right)$. At this point

$$
\dot{\hat{\phi}}=\dot{\theta}-\dot{\phi}-\omega=0,
$$

i.e., driven resonance between the $\mathrm{SAP}$ and the $s=n=1$ harmonic takes place, which we will refer to as Small-Action Resonance (SAR). It is clear that there is no threshold for perturbation amplitude for SAR to occur. The circle with the radius $\alpha /\left(2 \hat{\omega}_{\phi}\right)$ restricts the region where the particles are trapped with respect to the perturbation phase, which we will refer to as SmallAction Resonance Domain (SARD). The SARD, in fact, is produced by a simple shift of the vicinity of the O-point of the unperturbed system. Note that SARD typically arises in any integrable Hamiltonian system in the region of the phase space where, at least, one of the action variables is small [4].

The motion around SARD may result in radial redistribution of particles only in the case of fast switching on/off the perturbation. Otherwise, when the process begins and terminates slowly, the particles return almost to their initial radial positions after the perturbation disappears because the motion is adiabatic. Thus, the temporal evolution of the perturbation is crucial to determine the redistribution of SAP's due to their motion along SARD. In order to demonstrate this, let us estimate the displacement of a SAP caused by a $s=1$ perturbation which is sharply switched on and off, i.e., the characteristic time of the change of the perturbation amplitude is negligible in comparison with the period of the motion along SARD $\left(\tau_{S A R D}=2 \pi /\left(\omega_{b}-\omega_{\varphi}\right)\right)$. We find: 


$$
\frac{\Delta r}{\Delta r_{S A R D}} \sim \min \left\{1, \frac{\tau}{\tau_{S A R D}}\right\},
$$

where $\Delta r_{S A R D}=\left(2 / M \omega_{B}\right)^{1 / 2} \alpha /\left(\omega_{b}-\omega_{\varphi}\right)$ is the radial width of SARD, $\tau$ is the duration of the perturbation, $M$ is the particle mass. In another case, when $\alpha(t)=4 \alpha_{\max }(t / \tau)(1-t / \tau)$ and $\tau \gg \tau_{S A R D}$, we obtain

$$
\frac{\Delta r}{\Delta r_{S A R D}\left(\alpha_{\max }\right)} \leq \frac{2}{\pi} \frac{\tau_{S A R D}}{\tau}
$$

The presence of the $s=0$ harmonic changes the particle energy and results in an additional radial displacement, $\Delta r_{0}$. However, $\Delta r_{0}$ is small in comparison with $\Delta r_{S A R D}$. Furthermore, the $s=0$ harmonic does not lead to orbit transformation ( $J_{\theta}$ is not affected by this harmonic), and, as one can show, its effect on quasi-stagnation particles is even absent in the case of magnetic perturbations.

To study non-linear effects, we return to the Hamiltonian (1) and look for action-angle coordinates of the perturbed system. In other words, we proceed to the perturbed coordinate system centered in the SAR point, in which SAR does not exist. Considering the perturbation amplitude to be of the order of a small parameter $\varepsilon \ll 1$ and restricting ourselves to the case of $\omega=0$, we write $V=\varepsilon \sum_{s} \tilde{V}_{s}\left(J_{\theta}, J_{\phi}\right) \cos (s \theta-\phi)$. Then we use the perturbation technique [5] to find the canonical Lie transformation that annihilates the angle-dependent terms. To the first order in $\varepsilon$, the generator of the required transformation is produced by the following scalar generating function (the explicit expressions for the transformed coordinates in terms of the generating functions can be found in Refs. $[5,6])$ :

$$
S\left(J_{\theta}, J_{\phi}, \theta, \phi\right)=-\sum_{s} \frac{\tilde{V}_{s}}{s \omega_{\theta}-\omega_{\phi}} \sin (s \theta-\phi),
$$

where $\omega_{\theta}=\partial H^{(0)} / \partial J_{\theta}, \omega_{\phi}=\partial H^{(0)} / \partial J_{\phi}$. The denominators in Eq. (6) reflect the fact that the required transformation would not exist near resonances as the drift tori would be destroyed there. However, since $\omega_{\theta} \geq \omega_{\phi}$, such resonances do not occur. The Hamiltonian in the new coordinates takes the form

$$
\begin{aligned}
& H=H^{(0)}\left(J_{\theta}^{\prime}, J_{\phi}^{\prime}\right)+\varepsilon^{2}\left[f_{1}\left(J_{\theta}^{\prime}, J_{\phi}^{\prime}, \theta^{\prime}\right)\right. \\
& \left.+f_{2}\left(J_{\theta}^{\prime}, J_{\phi}^{\prime}, \theta^{\prime}\right) \cos \left(2 \phi^{\prime}\right)+f_{3}\left(J_{\theta}^{\prime}, J_{\phi}^{\prime}, \theta^{\prime}\right) \sin \left(2 \phi^{\prime}\right)\right]
\end{aligned}
$$

We see that the modes with $n=2$ and $n=0$ appear in the second order. The structure of the functions $f_{2}$ and $f_{3}$ (which are not presented here) shows that the non-linearity of the system and/or the presence of higher harmonics in $\theta$ (but not in $\vartheta$ ) are important for the $n=2$ harmonic to arise (otherwise, as we have shown above, the system is integrable). The procedure can be extended to next orders, where harmonics with $n>2$ are to appear.

Thus, the break of the toroidal symmetry produced by the $n=1$ mode of finite amplitude makes this mode to be non-monochromatic in the canonical toroidal angle of the perturbed system $\left(\phi^{\prime}\right)$. The appearance of higher harmonics in the perturbed system is of great importance because it makes possible multiple resonance islands due to interaction of SAP's with the $n=1$ perturbation through the resonances $s^{\prime} \omega_{\theta}^{\prime}=n^{\prime} \omega_{\phi}^{\prime}$, where $s^{\prime}$ and $n^{\prime}$ are the harmonic numbers in the perturbed system, which is likely to result in stochastic motion when the perturbation amplitude exceeds a certain magnitude.

In order to study this process, we generated numerically Poincaré maps of the particle motion in a tokamak in the presence of a steady-state $m=n=1$ perturbation, using the guiding-center code OFSEF [7]. We assumed that the perturbation was either magnetic, $\vec{B}=\nabla \varphi \times \nabla \Psi$, 
or electrostatic, $\vec{E}=\nabla \Phi$, with the potentials $\Psi$ and $\Phi$ given by $X=X_{\max } 4\left(r / r_{\max }\right)(1-$ $\left.r / r_{\max }\right) \exp (i \vartheta-i \varphi)$, where $X$ is either $\Psi$ or $\Phi, r_{\max }$ is the radius inside which the perturbation is localized, $X_{\max }$ is the maximum of $|X|$. The results for ${ }^{3} \mathrm{He}$ ions in a JET-like tokamak are presented in Figs. 1-4. In particular, Figs. 1-3 show the effect of an increase of the perturbation amplitude on the motion of $5-\mathrm{MeV}$ particles. We observe that when the amplitude is small (Fig. 1), the motion is regular with a small island chain $\left(n^{\prime}=3\right)$ in the potato region just outside SARD. At an intermediate amplitude, islands with $n^{\prime}=2$ and $n^{\prime}=3$ overlap, and a noticeable stochastic domain arises (Fig. 2). At a higher amplitude (Fig. 3), the stochasticity occupies the whole region of SAP's except for resonance islands, the largest of which are located near SAR; no stochasticity is seen in the intermediate region between SAP's and thin bananas (the periphery region in Fig. 3). The difference in the perturbation amplitude between Fig. 2, where the region of stochasticity is narrow, and Fig. 3, where the stochasticity dominates, is only by a factor of 1.5. Therefore, we conclude that there is an amplitude threshold of the appearance of the stochasticity.

To determine the dependence of the stochasticity threshold on the particle energy and tokamak parameters, we analyze the equations of the guiding-center motion. Assuming that the radial dependence of the perturbation is between $X \propto r^{0}$ and $X \propto r$, we find for the threshold amplitude:

$$
\Psi_{c r i t} \propto \rho^{\gamma}, \quad \Phi_{\text {crit }} \propto \rho^{4 / 3+\gamma},
$$

where $\gamma=2 / 3 \div 4 / 3$, the lower and upper limits corresponding to particles localized at $r \ll r_{\max }$ and $r \sim r_{\max }$, respectively. Using Eq. (8), one can predict that when, e.g., $\mathcal{E}=1 \mathrm{MeV}$ and the perturbation amplitude is the same as in Fig. 1, the picture is to be intermediate between Fig. 2 and Fig. 3 for $5-\mathrm{MeV}$ particles. The results of numerical calculations shown in Fig. 4 confirm this conclusion.

In the case when the magnetic perturbation is produced by a sawtooth crash in a JET-like tokamak, its amplitude corresponds to that in Figs. 1 and 4, which implies that motion of 1-MeV potatoes during the crash is stochastic, whereas that of $5-\mathrm{MeV}$ potatoes is regular. Calculations carried out for electrostatic perturbations with amplitudes corresponding to the crash show that such perturbations also lead to stochastic motion of $1-\mathrm{MeV}$ potatoes. Therefore, we conclude the crash results in stochastic motion of potato particles with $\mathcal{E} \lesssim 1 \mathrm{MeV}$.

The carried out analysis suggests two mechanisms of redistribution of stagnation particles (the motion around SARD and the stochasticity) due to the interaction with the $m=n=$ 1 harmonic. This provides new ways (in addition to those considered in Ref. [2,7]) to the explanation of disappearance of the hot-spot - a strongly localized neutron and $\gamma$-ray emitting region observed in experiments on JET [8] - during sawtooth crashes. Detailed analysis is required to determine which mechanisms dominate in the mentioned experiments.

In conclusion, we have shown that SAP's (potatoes, quasi-stagnation particles) can interact resonantly with an $n=1$ mode, which is often dominant in an MHD event. The finite perturbation amplitude leads to the appearance of higher toroidal harmonics (in the coordinate system associated with the perturbed particle motion), resulting in numerous resonance islands. When the perturbation amplitude exceeds the threshold magnitude, the stochasticity arises due to overlap of these islands, which is shown numerically for the $m=n=1$ mode. It is discovered that the stochasticity threshold is the lowest for potatoes and the highest for narrow bananas. The stagnation (and quasi-stagnation) particles are characterized by the intermediate threshold. In the case when the perturbation is associated with the sawtooth crash, the perturbation amplitude may be sufficient to result in stochasticity of the potato motion, but it is not sufficient to make the narrow-banana motion to be stochastic. When the perturbation is weak, no noticeable resonance islands exist. Then the $n=1$ mode leads mainly to regular particle 
motion around the helical stagnation orbit where SAR takes place. Quick switching on/off the perturbation may result in an essential particle redistribution, otherwise the redistribution is negligible because of adiabaticity of the process.

The research described in this publication was made possible in part by the Award No. UP2290 of the Government of Ukraine and CRDF. The authors thank V.S. Marchenko for discussions.

[1] R.B. White and M.S. Chance, Phys. Fluids 27, 2455 (1984).

[2] Ya.I. Kolesnichenko, V.V. Lutsenko, R.B. White, and Yu.V. Yakovenko, Phys. Plasmas 5, 2963 (1998).

[3] A.N. Kaufman, Phys. Fluids 15, 1063 (1972).

[4] Yu.V. Yakovenko, Ya.I. Kolesnichenko, V.V. Lutsenko, and R.B. White, "Small-action resonance in Hamiltonian systems and redistribution of energetic ions in tokamaks", PPPL Report 3349 (Princeton Plasma Physics Laboratory, 1999).

[5] R.G. Littlejohn, J. Math. Phys. 23 (1982) 742.

[6] A.J. Lichtenberg and M.A. Lieberman, Regular and stochastic motion (Springer-Verlag, Berlin - Heidelberg - New York, 1983).

[7] Ya.I. Kolesnichenko, V.V. Lutsenko, R.B. White, Yu.V. Yakovenko, and S.J. Zweben, Extended Synopses. 17th IAEA Fusion Energy Conference (Yokohama, 1998), 360 (IAEA, Vienna, 1998).

[8] O.N. Jarvis et al., Nucl. Fusion 36, 1513 (1996). 


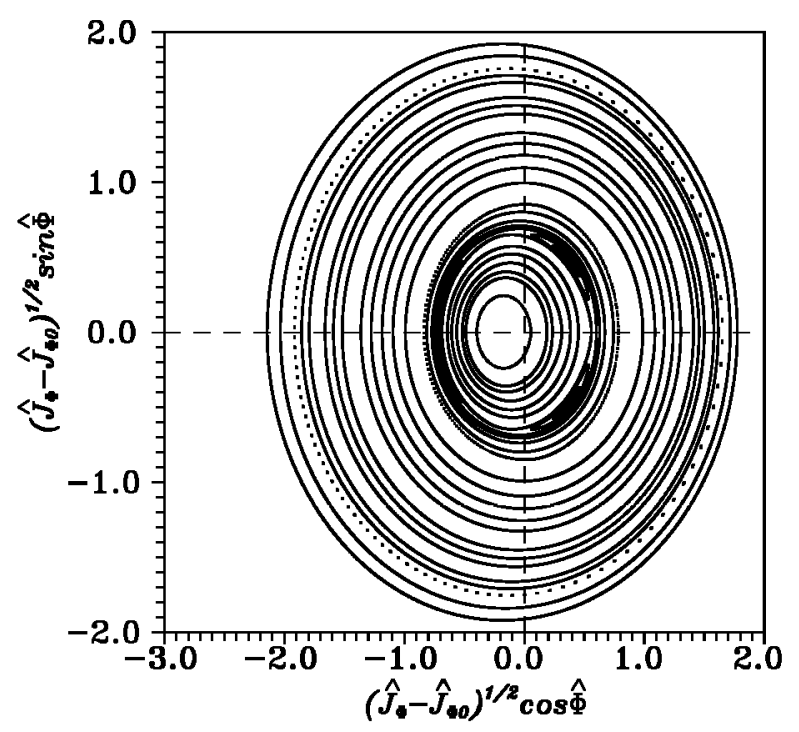

FIG. 1. The Poincaré map of the motion of $5-\mathrm{MeV}^{3} \mathrm{He}$ ions in a JET-like tokamak under the $m=n=1$ perturbation of the magnetic field with the normalized amplitude $\Psi_{\max }=0.1 \tilde{\Psi}$, where $\tilde{\Psi}=k B_{0} r_{\max }^{2} / 8, B_{0}=3.5 \mathrm{~T}$ is the equilibrium magnetic field, $k=1.2, r_{\max }=60 \mathrm{~cm}$. The points where particles cross the equatorial plane of the torus, moving downward, are presented.

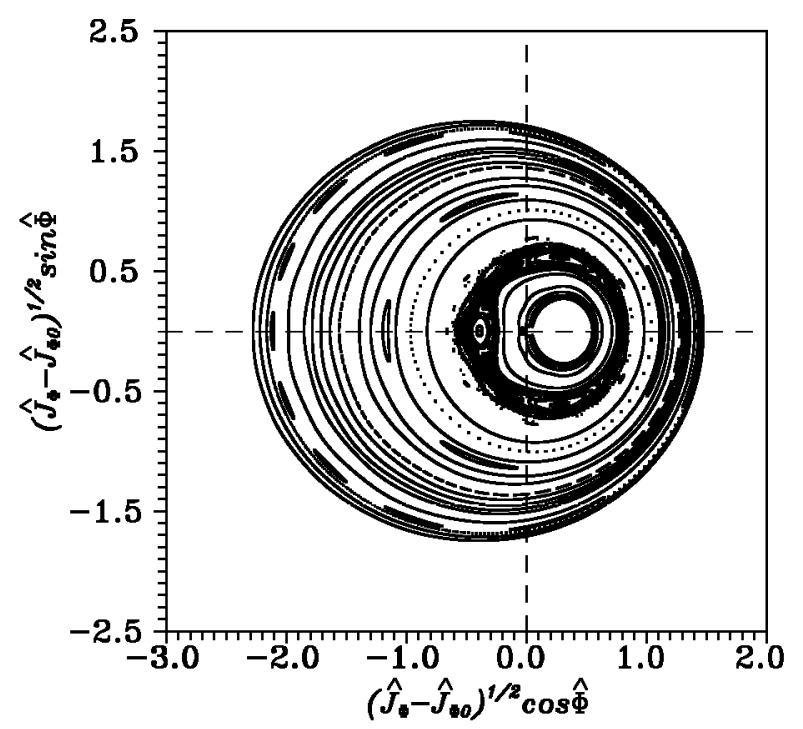

FIG. 2. The same as in Fig. 1 but for $\Psi_{\max }=0.2 \tilde{\Psi}$. 


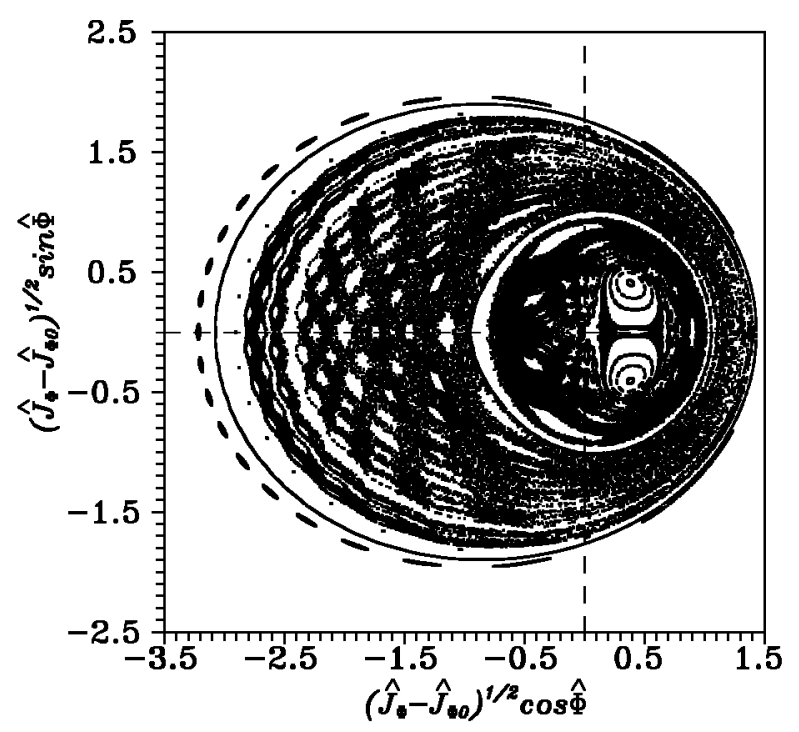

FIG. 3. The same as in Fig. 1 but for $\Psi_{\max }=0.3 \tilde{\Psi}$.

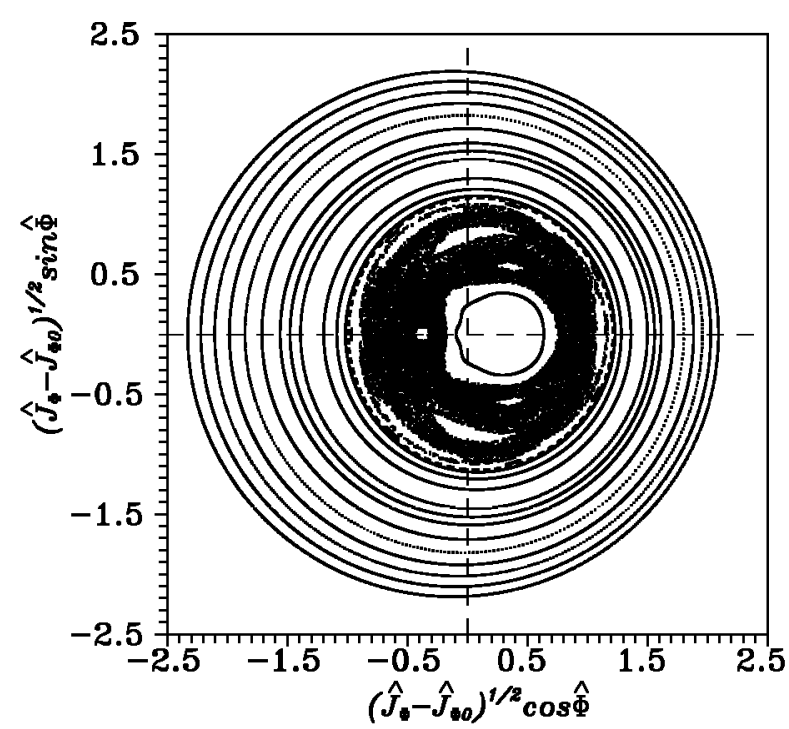

FIG. 4. The same as in Fig. 1 but for $\mathcal{E}=1 \mathrm{MeV}$. 